\title{
Rectal swabs as a viable alternative to faecal sampling for the analysis of gut microbiota functionality and composition
}

Benjamin H Mullish ( $\boldsymbol{D}$ b.mullish@imperial.ac.uk)

Imperial College London

Shiva T Radhakrishnan

St Mary's Hospital, Imperial College Healthcare NHS Trust

Kate I Gallagher

Imperial College London

Jose I Serrano-Contreras

Imperial College London

James L Alexander

Imperial College London

Jesus Miguens Blanco

Imperial College London

Nathan P Danckert

Imperial College London

Maria Valdivia-Garcia

Imperial College London

Billy J Hopkins

St Mary's Hospital, Imperial College Healthcare NHS Trust

Anik Ghai

London North-West Healthcare NHS trust

Jia V Li

Imperial College London

Julian R Marchesi

Imperial College London

Horace RT Williams

St Mary's Hospital, Imperial College Healthcare NHS Trust

\section{Research Article}

Keywords: gut microbiota, metabolome, swabs, 16S rRNA gene sequencing 
Posted Date: March 1st, 2022

DOI: https://doi.org/10.21203/rs.3.rs-1367190/v1

License: (c) (i) This work is licensed under a Creative Commons Attribution 4.0 International License. Read Full License

Version of Record: A version of this preprint was published at Scientific Reports on January 10th, 2023. See the published version at https://doi.org/10.1038/s41598-022-27131-9. 


\section{Abstract}

Faecal or biopsy samples are frequently used to analyse the gut microbiota, but issues remain with the provision and collection of such samples. Rectal swabs are widely-utilised in clinical practice and previous data demonstrate their potential role in microbiota analyses; however, studies to date have been heterogenous, and there is a particular lack of data concerning the utility of swabs for the analysis of the microbiota's functionality and metabolome. We compared paired stool and rectal swab samples from healthy individuals to investigate whether rectal swabs are a reliable proxy for faecal sampling. There were no significant differences in alpha and beta diversity measures between swab and faecal samples, and inter-subject variability was preserved. Additionally, no significant differences were demonstrated in abundance of major annotated phyla. Inferred gut functionality using Tax4Fun2 showed excellent correlation between the two sampling techniques (Pearson's coefficient $r=0.9217, P<0.0001$ ). Proton nuclear magnetic resonance ( ${ }^{1} \mathrm{H}$ NMR) spectroscopy enabled the detection of 20 metabolites with good correlation between rectal swab and faecal samples for butyrate, succinate and 5-aminovalerate relative abundances, though more variable degrees of association for other identified metabolites. These data support the utility of rectal swabs in both compositional and functional analyses of the gut microbiota.

\section{Introduction}

At present, analysis of the gut microbiota in humans primarily necessitates provision of a faecal sample or a mucosal biopsy. Current methods of faecal sampling are not without drawbacks; in particular, the very nature of defecation means that samples cannot be provided 'on demand' in a physician's office or to a research nurse and are reliant on appropriate collection by patients themselves. Faeces sampling may also present additional complexities, including the logistical challenges of having to transport samples between patient's home, the clinic and the laboratory, often with careful attention to maintaining cold chain conditions in the process ${ }^{1}$. Qualitative research demonstrates that patients are reluctant to handle their own stool and are embarrassed about transporting faeces ${ }^{2}$. Such aversion to traditional methods is mirrored in an inflammatory bowel disease (IBD) population, where compliance with stool collection to obtain faecal calprotectin measurements may be as low as $35 \%^{3}$. An alternative option to faecal sampling is colonic biopsy sampling at the time of an endoscopic procedure. In addition to the requirement for an invasive examination, endoscopic sampling frequently requires bowel preparation, which is well-recognised to affect intestinal microbiota composition ${ }^{4}$. As such, other options for obtaining samples to assess the human distal gut microbiota are of key interest.

Rectal swabs are widely-used in clinical practice; for example, national U.K guidance mandates their use for screening for intestinal colonization with carbapenemase-producing Enterobacteriaceae (CPE) for atrisk patients admitted to healthcare settings ${ }^{5}$. Studies of patient opinions demonstrate high levels of acceptability for CPE detection and rectal swabbing as a method of sample collection ${ }^{6}$. Given their acceptability, ease of administration, ease of self-administration and existing utility in clinical microbiology, rectal swabs might represent an attractive means for sampling the broader gut microbiota 
and aspects of the gut metabolome. However, the degree to which rectal swabs and faecal samples offer comparable assessment of the microbiota remains uncertain. Pilot studies have demonstrated close correlation between gut bacterial composition and alpha diversity in rectal swabs and matched faecal samples in both adult and pediatric populations ${ }^{7,8}$. An area of growing interest in this field relates to extending beyond profiling gut microbiota composition alone to also explore gut microbiota functionality; in particular, such "multi-omic" analyses are advocated to better delineate the interplay between microbe and human host ${ }^{9}$. One such key 'omics' technology is metabolomics, whereby advanced analytical chemistry techniques (including nuclear magnetic resonance spectroscopy (NMR) and mass spectroscopy (MS)) are used to identify and quantify small molecules within biofluids. ${ }^{1} \mathrm{H}$ NMR spectroscopy detects protons within small molecules and produces a spectrum related to proton profile within the biofluid, thereby having particular utility as a global metabolic profiling technique, including host- and microbe-derived metabolites ${ }^{10}$. Other attractions of ${ }^{1} \mathrm{H}$-NMR includes its high-throughput nature, its reproducibility, and that it is non-destructive to the samples analysed. Data have been published regarding optimised faecal collection and preparation for ${ }^{1} \mathrm{H}-\mathrm{NMR}$ analysis ${ }^{11}$; such data have confirmed the detection of a range of gut microbial metabolites of key interest to health and disease, and which give potential insight into gut microbiome-host interactions. As an example, ${ }^{1} \mathrm{H}$-NMR typically allows the detection of short chain fatty acids (SCFAs) ${ }^{11}$, metabolites important for gut health, with strong links to microbial metabolism of polysaccharides ${ }^{12}$. However, although metabolic profiling from swabs has been shown to be effective and achievable in vaginal swabs ${ }^{13}$, data are lacking for the detection of metabolites from rectal swabs.

In this study, we extend previous work comparing gut microbiota composition between paired faecal and swab samples to also explore the comparability of gut microbiota functionality, using both Tax4Fun2, a tool to infer microbial functionality using $16 \mathrm{~S}$ rRNA sequencing data and ${ }^{1} \mathrm{H}-\mathrm{NMR}$ spectroscopy-based metabolic profiling as our main tool of investigation.

\section{Methods}

\section{Study design and sample collection:}

The research and all associated experimental protocols was performed in accordance with approvals from the Research Governance and Integrity Team of Imperial College London, London, UK. Ethical approval for the study was granted by the Stanmore Research Ethics Centre approval (18/EM/0195), IRAS ref: 243310. All methods were carried out in accordance with relevant guidelines and regulations.

Matched faecal and rectal swab samples were obtained from 10 healthy individuals in a single center in London, UK. All participants gave informed consent to take part. All participants took no regular medication, were non-smokers, and had not used antibiotics for at least six weeks prior to donation. Whole faeces were collected in a faeces collector (FECOTAINER ${ }^{\circledR}$, AT Medical BV, The Netherlands) and COPAN FLOQSwabs ${ }^{\mathrm{TM}}$ (Copan Italia S.P.A., Brescia, Italy) were utilised as rectal swabs, given their 
previously demonstrated utility in faecal microbiota analysis ${ }^{14}$. The rectal swabs used were sterile with no preservative. Rectal swab collection was carried out at the same time as stool sample production and was obtained by self-insertion via the anus to a depth of $2-3 \mathrm{~cm}$ and rotated 3 times. Faecal samples and rectal swabs were stored at $-80^{\circ} \mathrm{C}$ as crude samples without the use of any cryopreservative until processed.

\section{S rRNA gene sequencing:}

DNA was extracted from crude faecal and swab samples using the DNeasy PowerLyzer PowerSoil Kit (Qiagen, Hilden, Germany) following manufacturer's instruction with the modification that samples were homogenised in a Bullet Blender Storm bead beater (Chembio, St Alban's, UK). DNA was quantified using a Qubit Fluorometer (ThermoFischer, UK), and was aliquoted and stored at $-80^{\circ} \mathrm{C}$ until ready for downstream use. Sample libraries were prepared following Illumina's 16S Metagenomic Sequencing Library Preparation Protocol ${ }^{15}$ using specifically designed V1/V2 hypervariable region primers ${ }^{16}$. Pooled sample library sequencing was performed using the Illumina MiSeq platform (Illumina Inc, Saffron Walden, UK) and the MiSeq Reagent Kit v3 (Illumina) using paired-end 300-bp chemistry. Processing of sequencing data was performed via the DADA2 pipeline (v1.18,) as previously described ${ }^{17}$, using the SILVA bacterial database Version 138 (https://www.arb-silva.de/ (accessed on $28^{\text {th }}$ July 2020)). In addition, 16S rRNA gene qPCR was performed to determine total bacterial biomass within each sample, using qPCR primers and protocol as previously described ${ }^{18}$.

A combination of $\mathrm{R}$ packages were used to analyse and visualise faecal/ swab microbiota sequencing data, including Phyloseq ${ }^{19}$, Vegan ${ }^{20}$, and ggplot $2^{21}$. Comparison of faecal and rectal swab microbiome taxonomy and ecological metrics was performed in R-studio (V1.2.5042). Shannon's diversity index, Inverse-Simpson's, Chao1 richness and Faith's phylogenetic diversity were used to calculate alphadiversity, while Aitchison's distance was used for beta-diversity analyses ${ }^{22}$ after center log-ratio data transformation (CLR) ${ }^{23}$; principal coordinates analyses ( $\left.P C O A\right)$ were generated to visualise the (dis)similarity between treatments and a permutational multivariate analysis of variance (PERMANOVA) statistically compared groupings within the data ${ }^{20}$. Extended error bar plots of taxonomic data were generated using the Statistical Analysis of Metagenomic Profiles (STAMP) software package using twosided White's nonparametric t-test with Benjamini-Hochberg FDR ${ }^{24}$. In addition, to putatively predict microbial functions from 16S rRNA gene sequencing data, the software Tax4fun2 v1.1.5 was used, with predicted relative values for different KEGG orthologues obtained ${ }^{25}$.

\section{Metabolomic profiling using ${ }^{1} \mathrm{H}-\mathrm{NMR}$ :}

\section{i. Sample preparation and data acquisition:}


Faecal water (FW) extracts for both faecal and rectal swab samples were obtained and analysed in $3.0 \mathrm{~mm}$ NMR tubes, as per previously described protocols ${ }^{11,26}$, with the additional step of vortexing/sonicating swabs (described further in Supplementary Methods). Rectal swabs and faecal sample extracts were analysed using a Bruker $600 \mathrm{MHz}$ AVANCE III NMR spectrometer at $300 \mathrm{~K}$. The 1D

${ }^{1} \mathrm{H}$ NMR spectra were acquired using a standard one-dimensional pulse sequence, with saturation of the water resonance (noesygppr1d pulse program) during both the relaxation delay ( $R D=4 \mathrm{~s}$ ) and mixing time $\left(t_{m}=10 \mathrm{~ms}\right)$. In total, 4 dummy scans, 128 scans and $64 \mathrm{~K}$ data points were collected. Further information regarding comprehensive NMR set up parameters for all spectra acquired can be found in the Supplementary Methods.

\section{ii. Metabolomic Data Processing:}

1D ${ }^{1} \mathrm{H}$-NMR spectra were processed using vendor software TopSpin v3.5 (Bruker) and were automatically phased, baseline corrected and referenced to TSP. Data were imported into MATLAB (2014a, MathWorks) and redundant spectral regions corresponding to residual water $\left(d^{1} \mathrm{H} 4.67-4.92\right), \operatorname{TSP}\left(\mathrm{d}^{1} \mathrm{H}-0.5-0.85\right)$, and noise ( $d^{1} \mathrm{H}$ 8.67-11.0) were removed. The spectra of swab blanks confirmed the presence of a poly(ethylene glycol) derivative, acetone, acetate, formate, ethanol, methanol, compounds related to plastics, and traces of lactate (Supplementary Table 2); therefore, their spectral regions were removed to ensure uniformity and reliability when comparing between the spectra of FW with those of swab samples. Data were normalised using probabilistic quotient normalization (PQN) to compensate for differences in concentration $^{27}$.

\section{iii. Identification of metabolites:}

Metabolite annotation was carried out using selective 1D TOCSY, 2D-NMR experiments, and correlation spectroscopy on $1 \mathrm{D}{ }^{1} \mathrm{H}-\mathrm{NMR}$ data $\mathrm{set}^{28}$. Internal and external databases such as the Human Metabolome Data Base (HMDB; http://hmdb.ca/) ${ }^{29}$ and/or the Biological Magnetic Resonance Data Bank (BMRB; http://www.bmrb.wisc.edu) were used for confirmation of assignments.

\section{iv. Data analysis:}

Correlation between relative units in matched faecal and swab samples of each KEGG orthologue (in the case of Tax4Fun2 data) and log-transformed metabolite values (in the case of ${ }^{1} \mathrm{H}$-NMR data) were analysed by Pearson's coefficient models (two-tailed analysis $p<0.05$ as cut off for significance); analysis was performed using GraphPad Prism version 9.1.2 (225) (GraphPad Software, San Diego, California USA).

\section{Results}




\section{The gut microbiota composition of matched faecal samples and rectal swabs is closely comparable:}

We firstly compared the $16 \mathrm{~S}$ rRNA gene sequencing profiles for matched faecal samples and rectal swabs, comparing them both in terms of ecological metrics (diversity, richness, etc) and specific profiles at different taxonomic levels.

The alpha (a)-diversity of all rectal swabs and faecal samples was analysed using a range of metrics; no statistically significant differences between values for swabs and faeces were found $(P>0.05$, KruskalWallis; Fig. 1A). Beta ( $\beta$ )-diversity showed expected inter-subject variability, but no statistically significant overall differences between rectal swabs and faecal samples ( $P=0.982$ PERMANOVA) (Fig. 1B). Of note, the major annotated bacterial phyla (including Firmicutes, Bacteroidetes and Proteobacteria) showed no statistically significant differences in relative abundance between swab and faecal samples $(q>0.05$, White's non-parametric two-sided t-test, Benjamini-Hochberg FDR correction; Fig. 1C). The only phylum showing significance between groups was Campilobacterota, which was enriched in swabs relative to faeces ( $q=0.007$; Supplementary Fig. 1A); however, this was only a feature in two participants, and made up $<10 \%$ of the overall reads in those participants. Similarly, only 5 out of 35 annotated bacterial families demonstrated statistically significant differences in relative abundance between faecal samples and matched swabs, with no such differences seen in the predominant families of Bacteroidaceae, Lachnospiraceae, Prevotellaceae, or Ruminococcaceae (Fig. 1D, Supplementary Fig. 1B). Interestingly, 16S rRNA gene copy number in the DNA extracted from samples was not found to be different between stool and swab ( $P>0.05$, Mann-Whitney, Fig. 1E). When 16S rRNA gene sequencing relative abundance data from samples was corrected for bacterial biomass as derived from 16S rRNA gene qPCR ${ }^{30}$, there again remained close comparability of profiles (Supplementary Fig. 2). These data build upon the conclusions from other studies that a rectal swab is an appropriate substitute for a faecal sample for profiling of gut microbiome composition.

\section{Matched faecal samples and rectal swabs demonstrate comparable functionality, in terms of both inferred function and metabolome:}

Given our particular interest in gut microbiota functionality, we went on to compare inferred functionality (using predicted relative values of KEGG orthologues, derived using Tax4Fun2), as established from matched rectal swabs and faecal samples. We observed very close correlation and excellent comparability of the data obtained using both sampling techniques, as analysed by Pearson's correlation $(r=0.9217, P<0.0001$; Fig. 2A).

We next investigated the degree to which metabolomic profiles obtained from faecal samples and matched rectal swabs using ${ }^{1} \mathrm{H}-\mathrm{NMR}$ were similar. Firstly, we used established NMR protocols ${ }^{26}$ to 
identify and quantify a range of different metabolites from spectral profiles that could be reliably recognised in both groups of samples, focusing on gut microbial metabolites or those with an association to host-microbial interactions; 20 such metabolites were identified (Supplementary Table 2). On correlation of all relative abundances together between faecal and swab samples for these identified metabolites, excellent correlation was found $(r=0.7779, P<0.0001$; Fig. 2B).

Further univariate analysis was performed on metabolites identified by ${ }^{1} \mathrm{H}-\mathrm{NMR}$ in both faeces and swabs, with a particular focus on those with a gut microbial origin. Specifically, we evaluated correlation between faecal and swab results for the identified short chain fatty acids; this analysis showed good correlation for butyrate $(r=0.6945, P=0.0258$; Fig. $3 A)$, but more modest correlation for propionate $(r=$ $0.5298, P=0.1152$; Fig. 3B). Furthermore, good correlation was seen for several metabolites closely associated with gut microbiome-host interactions, including succinate $(r=0.8945, P=0.0005$; Fig. 3C), 5 aminovalerate $(r=0.6816, P=0.003$; Fig. 3D), and phenylalanine $(r=0.6877, P=0.0279 ;$ Fig. 3E). However, the strength of correlation between swab and faecal data from other identified metabolites was more variable (Supplementary Fig. 3). Of note, a general pattern was observed for those of the annotated metabolites with higher overall relative values (particularly in swabs) being those with the strongest correlation between rectal and stool values.

\section{Discussion}

While colonic biopsies and faecal samples have a well-established role in profiling different aspects of the gut microbiota, both have drawbacks associated with their use. Clear attractions for the potential use of rectal swabs include the ease with which they can be administered and transported, and the high levels of acceptability to patients/ research study participants ${ }^{6}$. Earlier studies which compared rectal swabs to colonic biopsy samples did not demonstrate favorable correlation between the microbiota from the two communities ${ }^{7,31,32}$; however, mucosal microbiota samples in the studies were obtained after bowel purgatives with the authors acknowledging this limitation 7 . Additionally, rectal swabs may not obtain the same mucosal adherent microbiota as biopsy samples, which may explain the poor correlation between the sampling types ${ }^{33}$. Conversely, the same publications $s^{7,32}$ and recent work ${ }^{1,8,34-39}$ have demonstrated rectal swab microbiota communities to be closely related to matched faecal samples. Although there is heterogeneity between studies in terms of sample storage, populations sampled (healthy controls or disease) and microbial analysis techniques, studies tend to demonstrate with overall consistency that rectal swabs are a reliable proxy of faecal sampling for microbiota compositional analyses.

However, to date, previous publications using rectal swabs in gut microbiota research have mainly focused on the composition of the bacterial community, with data lacking with regards to the functionality of the microbiota and host interaction $17,8,31,32,34-39$. One study linked swab microbiota populations to gut microbiota functionality by interpreting KEGG pathways ${ }^{32}$, but generally there is a paucity of data with regards to profiling of microbiota functionality with rectal swabs. More specifically, 
there is a particular lack of data exploring the relationship between rectal swabs and stool for the assessment of other 'omic' profiles related to the microbiota, including the gut metabolome. In the current study, we used ${ }^{1} \mathrm{H}-\mathrm{NMR}$ (as a means of global metabolite profiling) to investigate this area, with our particular focus on metabolites related to host-microbiota interactions, given the growing interest of gut microbial metabolites to health and disease states, with one particular example of the latter being inflammatory bowel disease (IBD) ${ }^{40}$. The SCFAs butyrate and propionate are understood to be relevant to the pathogenesis of IBD, with previous work illustrating levels of these metabolites to be closely correlated to populations of Faecalibacterium prausnitzii and Roseburia hominis, bacteria which are known to be less abundant in active inflammation ${ }^{41}$. F. prausnitzii itself is a microbe of interest in IBD with a higher abundance noted in responders compared with non-responders to biologic medication ${ }^{42}$. Moreover, SCFAs themselves are thought to exert direct anti-inflammatory effects, such as inhibition of the pro-inflammatory cytokine tumor necrosis factor alpha (TNF-a) production from neutrophils ${ }^{43}$. Our research indicates that rectal swabs sample these SCFAs at comparable levels to corresponding faecal samples. Interestingly, our work also noted excellent correlation between both sample types in levels of other metabolites relevant to microbiome research, including succinate (a metabolite which has been implicated in fistulizing Crohn's disease (CD $)^{44}$ and is an important substrate to improve glucose homeostasis ${ }^{45}$ ), 5 -aminovalerate (which is associated with proline metabolism pathways ${ }^{46}$ ), and phenylalanine (an amino acid found to be enriched in the gut in $\mathrm{IBD}^{47}$ ). However, more variable levels of correlation were found for other annotated metabolites, particularly for those identified at lower relative values. One potential explanation for this is what may be expected intuitively regarding swab use, i.e., that biomass of material obtained by swab sampling may be a factor that influences the metabolite profile that may be obtained. Options to mitigate this issue may include using alternative swab designs that may facilitate collection of material, and/or the use of more than one swab per collection; however, as ever, such options have to be balanced against acceptability to patients, one of the major drivers towards consideration of swab use in the first place. Another possible explanation for any disparity between rectal and swab metabolic profiles may also represent the practicalities of sample handling. More specifically, previous work from our laboratory observed that, for ${ }^{1} \mathrm{H}-\mathrm{NMR}$ analysis of a faecal sample to be fully representative, the whole sample requires homogenization, to account for differences in metabolic profile on the surface versus within the stool, likely reflecting oxygen exposure and its impact upon stool microbe metabolism, and freezing within 4-6 hours, both of which may be cumbersome. By their nature ${ }^{11}$, rectal swabs require no initial sample handling phase and are easy to freeze, so may give a more representative simple 'snapshot' of the gut metabonome. Newer, reliable methods of stool sampling exist such as OMNIgene-GUT ${ }^{\circledR}$, which has good results in microbial DNA analyses ${ }^{48}$; further recent work demonstrates good correlation between selected bile acids as detected in crude stool and via collection using OMNIgene-GUT ${ }^{\circledR}$, but with a significantly reduced concentration of total bile acids using this kit compared to faecal sampling ${ }^{49}$.

Whilst our results are promising, our study does have limitations, and further work would be required before utilizing rectal swabs more broadly as a tool to study the gut metabolome instead of stool. We 
recruited a relatively small number of participants, and trends of the abundance of some metabolites (notably propionate) may have been significant in a larger population. Our collection from healthy individuals allowed for snap freezing and prompt storage of samples, which may not always be feasible if swabs are utilised in a clinical outpatient setting. Of interest, even though overall metabolite correlation between swabs and faeces was very good, certain individual metabolites did not demonstrate such strong correlation; whether this represents the limit of detection of ${ }^{1} \mathrm{H}-\mathrm{NMR}$, the volatility of particular metabolites, or other factors requires further exploration. Use of other high sensitivity metabolomics pipelines - including mass spectrometry techniques - may be more appropriate for particular metabolite groups. Overall, it can be inferred that certain metabolites may be less detectable by rectal swabs, but more data in a larger population are required.

\section{Conclusion}

While several early studies in different settings have suggested that rectal swabs may have utility in identifying gut microbiota composition comparable to faecal samples, the data regarding their use as a tool in identifying the gut metabolome remain more limited. This question is particularly pertinent given the growing role of omic studies - including those focused on gut microbial metabolites - as a route to exploring gut microbiome-host interactions. In this study, we use ${ }^{1} \mathrm{H}-\mathrm{NMR}$ (as a global metabolic profiling modality) to demonstrate that rectal swabs show promise as a tool to analyse both the gut microbial functionality (including the metabolome) and bacterial compositional profile with comparable efficacy to faecal samples, but that further method development is required before they might be suitable for more widespread clinical adaptation.

\section{Declarations}

\section{Acknowledgements:}

BHM is the recipient of an NIHR Academic Clinical Lectureship (CL-2019-21-002). JLA is the recipient of an NIHR Academic Clinical Lectureship (CL-2019-21-502), funded by Imperial College London and The Joyce and Norman Freed Charitable Trust. JVL is supported by the Medical Research Council (MRC) New Investigator Research Grant (MR/P002536/1) and ERC Starting Grant (715662). The Division of Digestive Diseases at Imperial College London receives financial and infrastructure support from the NIHR Imperial Biomedical Research Centre (BRC) based at Imperial College Healthcare NHS Trust and Imperial College London.

\section{Author contributions:}

STR, BHM, JLA, JVL, JRM and HRTW designed the study; STR, BHM, JLA, BJH and AG obtained regulatory approval and collected samples; JMB and NPD undertook microbiome analysis; KIG, JIS-C, 
MV-G and JVL undertook metabolomic analysis; STR, BHM, JLA, JMB, NPD, JRM and HRTW wrote the first draft of the manuscript; all authors contributed to writing of and approved the final manuscript.

\section{Data Availability:}

Sequencing data from this study (in fastq-format) are publicly available for download at the European Nucleotide Archive (ENA) database using study accession number PRJEB50814 (http://www.ebi.ac.uk/ena/data/view/PRJEB50814). The datasets used and/or analysed during the current study are available from the corresponding author on reasonable request.

\section{Additional information:}

STR has received conference sponsorship from Vifor. JLA has received conference sponsorship from Vifor. JRM has been paid for consultancy by Cultech Ltd and Enterobiotix Ltd. All other authors declare no competing interests.

\section{References}

1. Williams, G. M.et al. Gut microbiome analysis by post: Evaluation of the optimal method to collect stool samples from infants within a national cohort study. PLoS One 14, e0216557, doi:10.1371/journal.pone.0216557 (2019).

2. Lecky, D. M., Hawking, M. K., McNulty, C. A. \& group, E. s. Patients' perspectives on providing a stool sample to their GP: a qualitative study. Br J Gen Pract 64, e684-693, doi:10.3399/bjgp14X682261 (2014).

3. Marechal, C.et al. Compliance with the faecal calprotectin test in patients with inflammatory bowel disease. United European Gastroenterol J 5, 702-707, doi:10.1177/2050640616686517 (2017).

4. Jalanka, J.et al. Effects of bowel cleansing on the intestinal microbiota. Gut 64, 1562-1568, doi:10.1136/gutjnl-2014-307240 (2015).

5. HPS. Toolkit for the early detection, management and control of carbapenemase-producing Enterobacteriaceae in Scottish acute settings. (2016).

6. Currie, K.et al. The acceptability of screening for Carbapenemase Producing Enterobacteriaceae (CPE): cross-sectional survey of nursing staff and the general publics' perceptions. Antimicrob Resist Infect Control 7, 144, doi:10.1186/s13756-018-0434-x (2018).

7. Budding, A. E.et al. Rectal swabs for analysis of the intestinal microbiota. PLoS One 9, e101344, doi:10.1371/journal.pone.0101344 (2014).

8. Reyman, M., van Houten, M. A., Arp, K., Sanders, E. A. M. \& Bogaert, D. Rectal swabs are a reliable proxy for faecal samples in infant gut microbiota research based on 16S-rRNA sequencing. Sci Rep 9, 16072, doi:10.1038/s41598-019-52549-z (2019). 
9. Lamichhane, S., Sen, P., Dickens, A. M., Oresic, M. \& Bertram, H. C. Gut metabolome meets microbiome: A methodological perspective to understand the relationship between host and microbe. Methods 149, 3-12, doi:10.1016/j.ymeth.2018.04.029 (2018).

10. Nicholson, J. K. \& Lindon, J. C. Metabonomics. Nature 455, 1054-1056 (2008).

11. Gratton, J.et al. An optimised sample handling strategy for metabolic profiling of human faeces. Anal Chem 88, 4661-4668, doi:10.1021/acs.analchem.5b04159 (2016).

12. Miller, T. L. \& Wolin, M. J. Pathways of Acetate, Propionate, and Butyrate Formation by the Human Faecal Microbial Flora. APPLIED AND ENVIRONMENTAL MICROBIOLOGY 62, 1589-1592, doi:https://doi.org/10.1128/aem.62.5.1589-1592.1996 (1996).

13. Pruski, P.et al. Medical Swab Analysis Using Desorption Electrospray lonization Mass Spectrometry: A Noninvasive Approach for Mucosal Diagnostics. Anal Chem 89, 1540-1550, doi:10.1021/acs.analchem.6b03405 (2017).

14. Tedjo, D. I.et al. The effect of sampling and storage on the faecal microbiota composition in healthy and diseased subjects. PLoS One 10, e0126685, doi:10.1371/journal.pone.0126685 (2015).

15. Amplicon, P., Clean-Up, P. \& Index, P. 16s metagenomic sequencing library preparation. Illumina. com. (2013).

16. Mullish, B. H.et al. Functional microbiomics: Evaluation of gut microbiota-bile acid metabolism interactions in health and disease. Methods 149, 49-58, doi:10.1016/j.ymeth.2018.04.028 (2018).

17. Callahan, B. J.et al. DADA2: High-resolution sample inference from Illumina amplicon data. Nat Methods 13, 581-583, doi:10.1038/nmeth.3869 (2016).

18. Mullish, B. H.et al. Microbial bile salt hydrolases mediate the efficacy of faecal microbiota transplant in the treatment of recurrent Clostridioides difficile infection. Gut 68, 1791-1800, doi:10.1136/gutjnl2018-317842 (2019).

19. McMurdie, P. J. \& Holmes, S. phyloseq: an R package for reproducible interactive analysis and graphics of microbiome census data. PLoS One 8, e61217, doi:10.1371/journal.pone.0061217 (2013).

20. Oksanen, J.et al. Package 'vegan', <https://cran.r-project.org, https://github.com/vegandevs/vegan> (2020).

21. Wickham, H. ggplot2Elegant Graphics for Data Analysis. Second edn, (Springer Nature, 2016).

22. Aitchison, J. The Statistical Analysis of Compositional Data. Journal of the Royal Statistical Society: Series B (Methodological) 44, 139-160, doi: https://doi.org/10.1111/j.2517-6161.1982.tb01195.x (1982).

23. Gloor, G. B., Macklaim, J. M., Pawlowsky-Glahn, V. \& Egozcue, J. J. Microbiome Datasets Are Compositional: And This Is Not Optional. Front Microbio/ 8, 2224, doi:10.3389/fmicb.2017.02224 (2017).

24. Parks, D. H., Tyson, G. W., Hugenholtz, P. \& Beiko, R. G. STAMP: statistical analysis of taxonomic and functional profiles. Bioinformatics 30, 3123-3124, doi:10.1093/bioinformatics/btu494 (2014). 
25. Wemheuer, F.et al. Tax4Fun2: prediction of habitat-specific functional profiles and functional redundancy based on 16 S rRNA gene sequences. Environ Microbiome 15, 11, doi:10.1186/s40793020-00358-7 (2020).

26. Dona, A. C.et al. Precision high-throughput proton NMR spectroscopy of human urine, serum, and plasma for large-scale metabolic phenotyping. Anal Chem 86, 9887-9894, doi:10.1021/ac5025039 (2014).

27. Dieterle, F., Ross, A., Schlotterbeck, G. \& Senn, H. Probabilistic Quotient Normalization as Robust Method to Account for Dilution of Complex Biological Mixtures. Application in 1H NMR Metabonomics. Anal Chem 78, 4281-4290, doi:https://doi.org/10.1021/ac051632c (2006).

28. Posma, J. M.et al. Subset optimization by reference matching (STORM): an optimised statistical approach for recovery of metabolic biomarker structural information from $1 \mathrm{H}$ NMR spectra of biofluids. Anal Chem 84, 10694-10701, doi:10.1021/ac302360v (2012).

29. Wishart, D. S.et al. HMDB 4.0: the human metabolome database for 2018. Nucleic Acids Res 46, D608-D617, doi:10.1093/nar/gkx1089 (2018).

30. Jian, C., Luukkonen, P., Yki-Jarvinen, H., Salonen, A. \& Korpela, K. Quantitative PCR provides a simple and accessible method for quantitative microbiota profiling. PLoS One 15, e0227285, doi:10.1371/journal.pone.0227285 (2020).

31. Araujo-Perez, F.et al. Differences in microbial signatures between rectal mucosal biopsies and rectal swabs. Gut Microbes 3, 530-535, doi:10.4161/gmic.22157 (2012).

32. Jones, R. B. et al. Inter-niche and inter-individual variation in gut microbial community assessment using stool, rectal swab, and mucosal samples. Sci Rep 8, 4139, doi:10.1038/s41598-018-22408-4 (2018).

33. Shen, T. D.et al. The Mucosally-Adherent Rectal Microbiota Contains Features Unique to AlcoholRelated Cirrhosis. Gut Microbes 13, 1987781, doi:10.1080/19490976.2021.1987781 (2021).

34. Bassis, C. M.et al. Comparison of stool versus rectal swab samples and storage conditions on bacterial community profiles. BMC Microbio/17, 78, doi:10.1186/s12866-017-0983-9 (2017).

35. Bokulich, N. A., Maldonado, J., Kang, D. W., Krajmalnik-Brown, R. \& Caporaso, J. G. Rapidly Processed Stool Swabs Approximate Stool Microbiota Profiles. mSphere 4, doi:10.1128/mSphere.00208-19 (2019).

36. Biehl, L. M.et al. Usability of rectal swabs for microbiome sampling in a cohort study of hematological and oncological patients. PLoS One 14, e0215428, doi:10.1371/journal.pone.0215428 (2019).

37. Fair, K. et al. Rectal Swabs from Critically III Patients Provide Discordant Representations of the Gut Microbiome Compared to Stool Samples. mSphere 4, doi:10.1128/mSphere.00358-19 (2019).

38. Short, M. I.et al. Comparison of rectal swab, glove tip, and participant-collected stool techniques for gut microbiome sampling. BMC Microbio/ 21, 26, doi:10.1186/s12866-020-02080-3 (2021).

39. Mazzarelli, A.et al. 16S rRNA gene sequencing of rectal swab in patients affected by COVID-19. PLoS One 16, e0247041, doi:10.1371/journal.pone.0247041 (2021). 
40. Lloyd-Price, J.et al. Multi-omics of the gut microbial ecosystem in inflammatory bowel diseases. Nature 569, 655-662, doi:10.1038/s41586-019-1237-9 (2019).

41. Machiels, K.et al. A decrease of the butyrate-producing species Roseburia hominis and Faecalibacterium prausnitzii defines dysbiosis in patients with ulcerative colitis. Gut $63,1275-1283$, doi:10.1136/gutjnl-2013-304833 (2014).

42. Radhakrishnan, S. T.et al. Systematic review: the association between the gut microbiota and medical therapies in inflammatory bowel disease. Aliment Pharmacol Ther 55, 26-48, doi:10.1111/apt.16656 (2022).

43. Vinolo, M. A. et al. Suppressive effect of short-chain fatty acids on production of proinflammatory mediators by neutrophils. J Nutr Biochem 22, 849-855, doi:10.1016/j.jnutbio.2010.07.009 (2011).

44. Ortiz-Masia, D.et al. Succinate Activates EMT in Intestinal Epithelial Cells through SUCNR1: A Novel Protagonist in Fistula Development. Cells 9, doi:10.3390/cells9051104 (2020).

45. De Vadder, F.et al. Microbiota-Produced Succinate Improves Glucose Homeostasis via Intestinal Gluconeogenesis. Cell Metab 24, 151-157, doi:10.1016/j.cmet.2016.06.013 (2016).

46. Bisht, V.et al. Integration of the Microbiome, Metabolome and Transcriptomics Data Identified Novel Metabolic Pathway Regulation in Colorectal Cancer. Int J Mol Sci 22, doi:10.3390/ijms22115763 (2021).

47. Bosch, S.et al. Faecal Amino Acid Analysis Can Discriminate De Novo Treatment-Naive Pediatric Inflammatory Bowel Disease From Controls. J Pediatr Gastroenterol Nutr 66, 773-778, doi:10.1097/MPG.0000000000001812 (2018).

48. Neuberger-Castillo, L.et al. Method Validation for Extraction of DNA from Human Stool Samples for Downstream Microbiome Analysis. Biopreserv Biobank 18, 102-116, doi:10.1089/bio.2019.0112 (2020).

49. Neuberger-Castillo, L., Ammerlaan, W. \& Betsou, F. Fitness for purpose of stabilised stool samples for bile acid metabolite analyses. Sci Rep 11, 7904, doi:10.1038/s41598-021-86784-0 (2021).

\section{Figures}


A

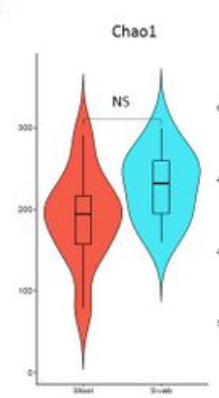

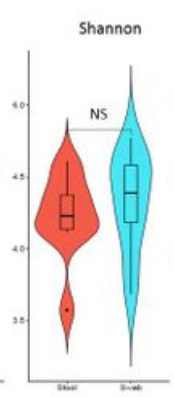

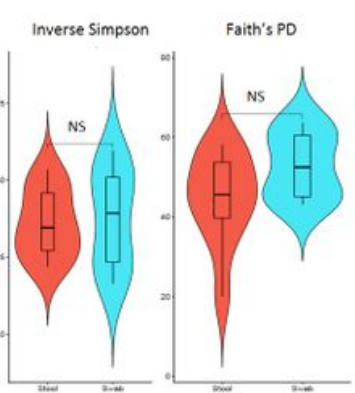

C

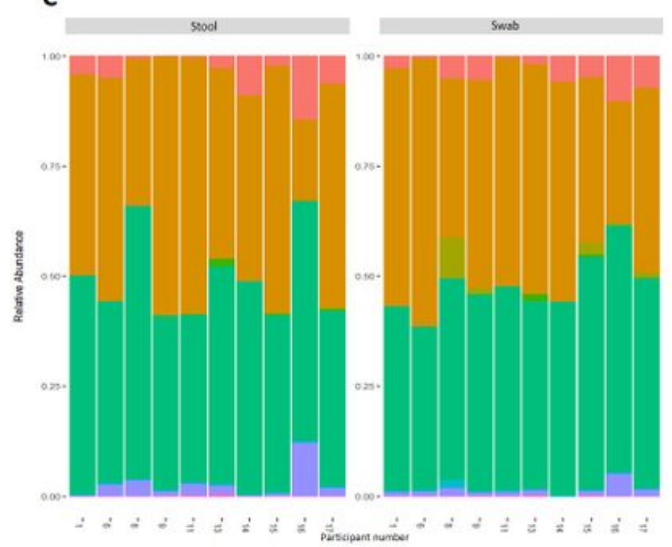

D

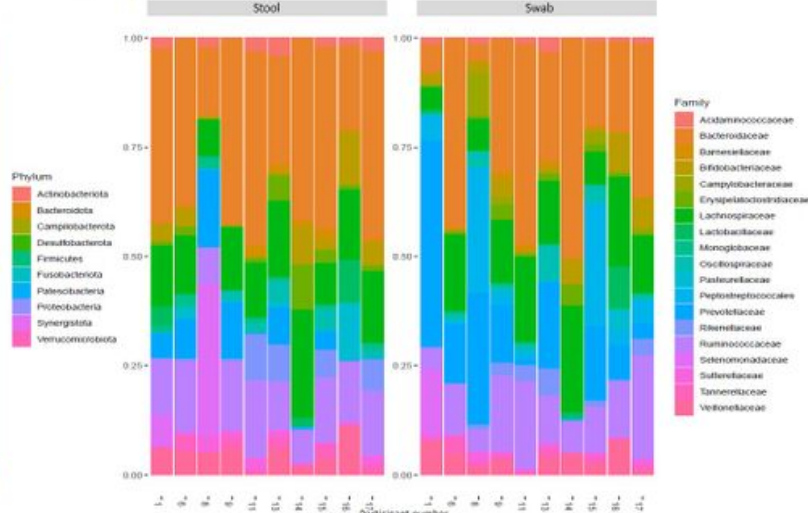

B

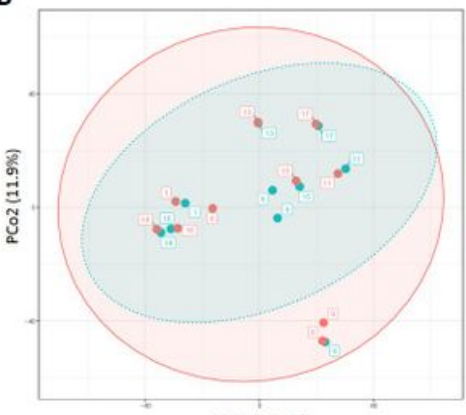

PCO1 (12.1\%)

E

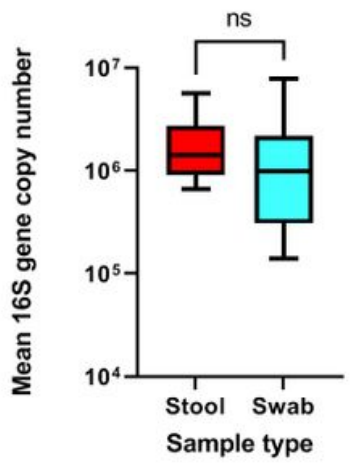

Figure 1

Comparison of compositional analysis of the gut microbiome, as assessed by matched faeces vs rectal swabs. A) Alpha diversity metrics (Kruskal-Wallis); B) Beta diversity, as represented by PCoA (numbers on points within figure represent study participant number); C) Relative abundance plots of all bacterial phyla; D) Relative abundance plot of major bacterial families (filtering used to remove families present at $<5 \%$ relative abundance; numbers on horizontal axis represent study participant number); E) $16 \mathrm{~S}$ rRNA gene copy number. Faeces: $n=10$ samples; swabs: $n=10$ samples.

A

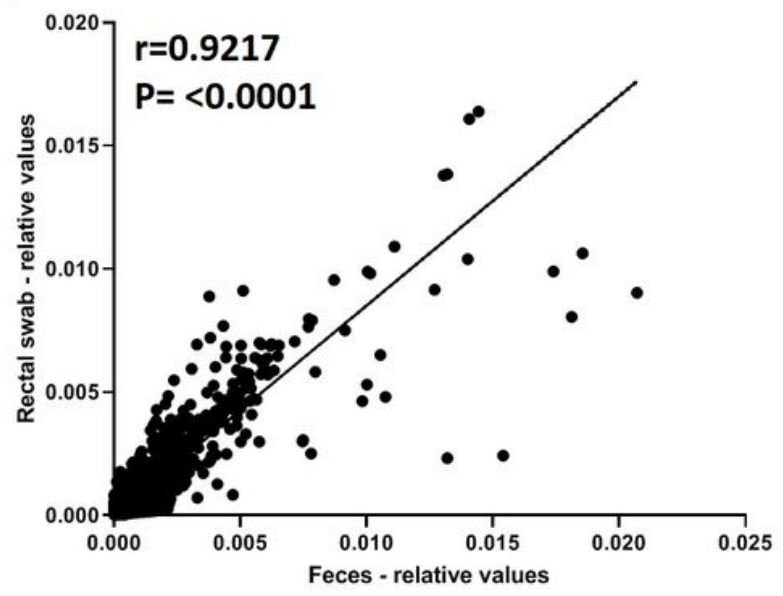

B

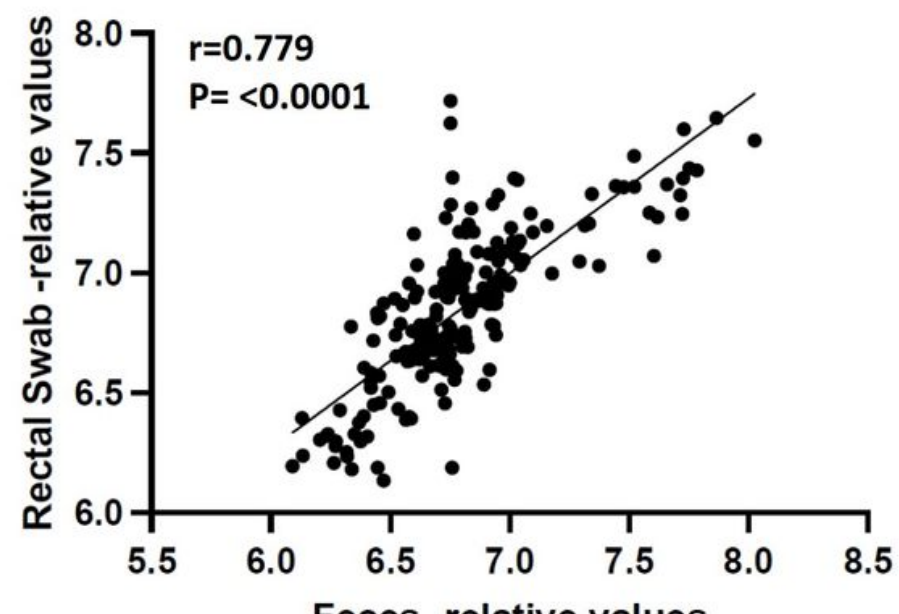

Feces-relative values 
Figure 2

Comparison of gut microbial functionality in matched faeces vs rectal swabs. A) Correlation of swab and faecal KEGG orthologue data obtained from Tax4Fun2, quantified as relative units (performed using Pearson's coefficient); B) correlation of all relative abundance values for identified metabolites from faecal and swab samples (performed using Pearson's coefficient). Faeces: $n=10$ samples; swabs: $n=10$ samples.

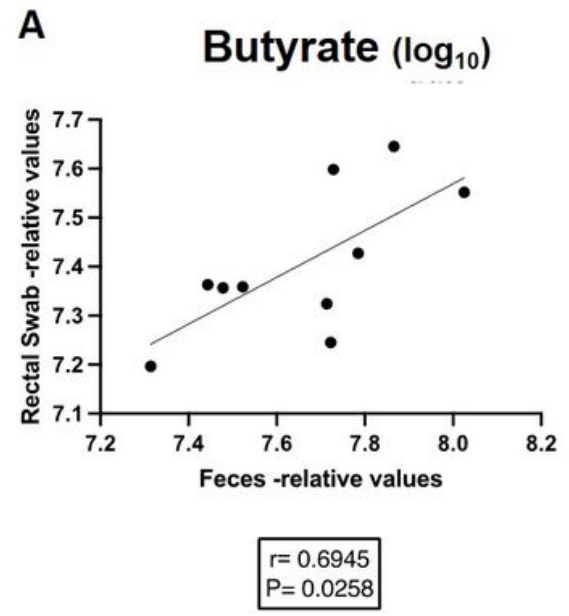

D

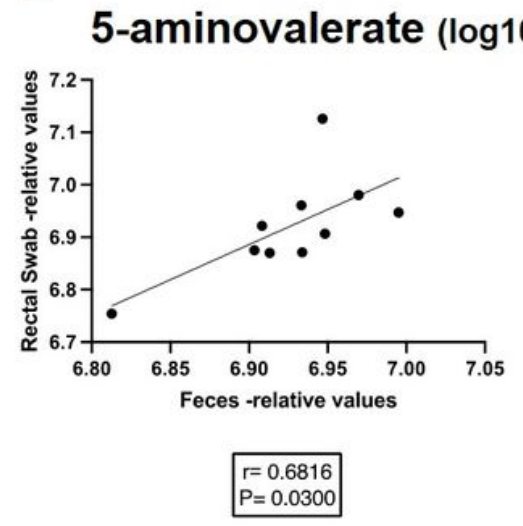

-aminovalerate $(\log 10)$
B

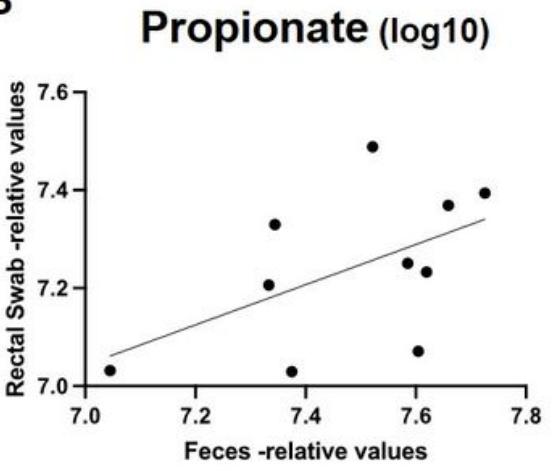

$r=0.5298$

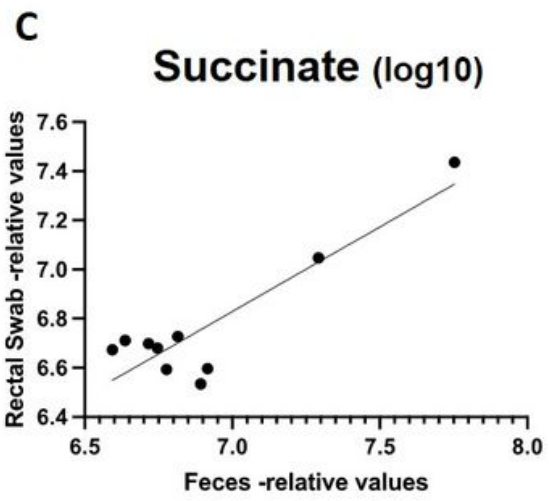

$r=0.8945$ $\mathrm{P}=0.0005$
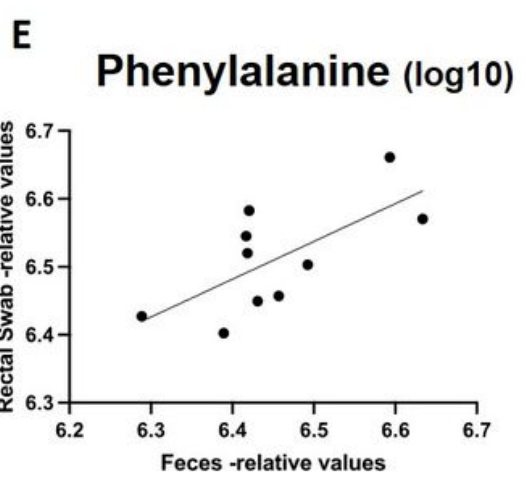

$r=0.6877$

$\mathrm{P}=0.0279$

Figure 3

Correlation of levels of selected gut microbial-related metabolites in rectal swabs and matched stool samples. A) butyrate; B) propionate; C) succinate; D) 5-aminovalerate; E) phenylalanine. Faeces: $n=10$ samples; swabs: $n=10$ samples.

\section{Supplementary Files}


This is a list of supplementary files associated with this preprint. Click to download.

- v25Supplementarydata07012022.docx 